\title{
Voltage tunable multiple quantum well distributed feedback filter with an electron beam written Schottky grating
}

\author{
O. Zia, P. K. Bhattacharya, J. Singh, and T. Brock \\ Solid State Electronics Laboratory, Department of Electrical Engineering and Computer Science, \\ University of Michigan, Ann Arbor, Michigan 48109-2122
}

(Received 21 February 1994; accepted for publication 1 May 1994)

\begin{abstract}
A novel optoelectronic filter with voltage-tunable transfer characteristics has been developed and implemented in a multiquantum well waveguide device. By virtue of the quantum-confined Stark effect, the refractive index in quantum wells at the periphery of a guiding region can be given a periodicity in the guiding direction by application of a bias on an electron-beam patterned Schottky gratïng atop the guide. If the period of the Schottky grating and associated index profile satisfies the Bragg condition, as in a resonant distributed feedback structure, band-reject filtering results. Altering the bias on the Schottky grating changes the refractive index in the wells, thereby providing tunability of the wavelength at which Bragg diffraction occurs.
\end{abstract}

Distributed feedback (DFB) structures have been extensively studied for use in high bandwidth communications systems where spectral purity and tunability of optical sources are required. ${ }^{1-3}$ Defined by holographic techniques or electron-beam lithography, these periodic index grating structures have been shown to produce transfer curves of extremely narrow linewidth. DFBs have been predominantly formed by an etch and regrowth process to form the periodic index grating. Tunability of the grating, as has been demonstrated in DFB lasers, can then be achieved by altering the carrier density in the grating region, thus changing the refractive index and the associated resonance wavelength of the DFB structure. ${ }^{4,5}$ Regrowth, however, is currently a low yield processing step, and carrier denisity tuning has speed limitations based on carrier recombination times. Such limitations inhibit these devices from taking a serious role in high speed tuning applications for state-of-the-art communications systems. By taking advantage of the enhanced Stark effect, we have been able to form a significant spatial index grating just under the top surface of a semiconductor by depositing a periodic Schottky metal grating on the surface. This index grating is formed by the field due to the Schottky barrier height. Tunability proceeds as follows: with the application of a reverse bias to the grating, we change the optical path length $L_{o p}$ of the grating period due to an imposed $\Delta n$ in the structure. Varying $\Delta n$, we change the wavelength at which the Bragg condition is met. By incorporating this structure into a waveguide, with the grating defined by the electron-beam lithography transverse to the guiding direction, a potentially high-speed tunable reflection filter is achieved without any regrowth steps.

The refractive index in quantum wells changes with applied bias by the same mechanism that the absorption changes, the quantum-confined Stark effect (QCSE). The QCSE is well understood and several electroabsorption devices and architectures have been achieved by employing it. ${ }^{6-8}$ Electrorefraction in quantum wells can be quantified using the Kramers-Kronig relations and is seen to have a quadratic dependence on transverse applied fields operating near the excitonic band edge. ${ }^{9,10}$ This enhanced electrorefractive effect, however, is accompanied by large absorption at the band edge. For DFB devices, which need long interaction lengths between the forward and backward coupled modes, the large absorption can be detrimental. Thus, the choice of an operating wavelength and design of the quantum wells are done to minimize absorption while still utilizing the large electrorefractive effects in quantum wells. Requiring a $\Delta n$ of at least 0.006 , giving a $10 \AA$ shift in the resonance wavelength, as a design constraint, a Kramers-Kronig optimization analysis suggests the design of the grating resonance at $50 \mathrm{meV}$ lower than the zero field heavy-hole excitonic transition in order to keep absorption acceptably low.

The device structure is grown by MBE on an $n^{+}$GaAs substrate and is shown in Fig. 1. A Schottky metal consisting of $250 \AA$ titanium and $1000 \AA$ gold is deposited on the AlGaAs cap layer. This metal constitutes a 128 period grating with a $0.36 \mu \mathrm{m}$ pitch and individual finger length of 5 $\mu \mathrm{m}$. It is formed by standard electron beam lithography and lift off techniques. A $3 \mu \mathrm{m}$ wide ridge waveguide is optically defined to pass under and perpendicular to the Schottky grating. The ridge is dry etched to be $0.9 \mu \mathrm{m}$ deep using a reactive ion etching (RIE) process. The dry etching process undercuts the area between the guide and contact pad mesa forming an airbridge to a contact pad (Fig. 2). A $0.7 \mu \mathrm{m}$ thick plasma-enhanced chemical vapor deposition (PECVD) of $\mathrm{SiO}_{2}$ is then done to both passivate the waveguide between grating fingers and protect the surface. Contact holes to the Schottky grating strips are then opened by using RIE and a thick interconnect layer of $\mathrm{Ti} / \mathrm{Al} / \mathrm{Ti} / \mathrm{Au}$ makes the probing pad for the Schottky contact. The wafer is then thinned to a thickness of $100 \mu \mathrm{m}$ and backside $n$-type ohmic contact is formed. The waveguide is then cleaved to $400 \mu \mathrm{m}$ length for optical measurements.

The diode characteristics of the device were first measured and exhibit a forward turn on voltage of $1.5 \mathrm{~V}$ and reverse breakdown voltage of $-16 \mathrm{~V}$. Photoluminescence data showed the heavy-hole excitonic peak of the wells to be at $845 \mathrm{~nm}$. Our targeted $0.36 \mu \mathrm{m}$ grating pitch, designed to achieve a second-order resonance $50 \mathrm{meV}$ below the absorption peak, was measured to be resonant at $861.7 \mathrm{~nm}$, rather than the desired $875 \mathrm{~nm}$. The corresponding $16 \mathrm{meV}$ error lies within the tolerance limits of the processing. The wave- 


\begin{tabular}{ccc}
\hline $200 \AA$ & $\mathrm{Al}_{0.3} \mathrm{Ga}_{0.7} \mathrm{As}$ & $\mathrm{n}=1.0 \times 10^{16} \mathrm{~cm}^{-3}$ \\
\hline 3 prd & $\mathrm{GaAs}^{\mathrm{A} s} / \mathrm{Al}_{0.3} \mathrm{Ga} 0.7 \mathrm{As}$ & \\
& $L_{W}=L_{B}=90 \AA$ & undoped \\
\hline $0.3 \mu \mathrm{m}$ & $\mathrm{Al}_{0.1} \mathrm{Ga}_{0.9} \mathrm{As}$ & undoped \\
\hline $0.5 \mu \mathrm{m}$ & $\mathrm{Al}_{0.3} \mathrm{Ga}_{0.7} \mathrm{As}$ & $\mathrm{n}=5.0 \times 10^{18} \mathrm{~cm}^{-3}$ \\
\hline \multicolumn{3}{c}{$\mathrm{n}+\mathrm{GaAs}$ Substrate }
\end{tabular}

FIG. 1. Waveguiding material structure as grown by MBE.

guide filters were measured using a temperature tunable GaAs/AlGaAs laser source. The source light was collimated and focused onto the waveguide and the output light was detected by a broad area silicon photodiode. Mode calculations indicate that just the tail of the mode, a mere $2.8 \%$ of the power, lies within the MQW region. Due to the proximity of the wells to the semiconductor surface, this number must be low in order to minimize radiation loss and loss due to absorption by the Schottky metal. Despite the almost negligible overlap of the optical mode with the wells, electroabsorption due to the wells is very strong as can be seen in Fig. 3. This strong interaction indicates the possibility of a significant coupling coefficient for DFB action and provides a basis for this work.

The relative output intensity was subsequently measured for a variety of biases across the device and wavelengths and is shown in Fig. 4. From the data it is clear that a resonance can be seen at zero bias at $861.7 \mathrm{~nm}$ by a dip in the output intensity. The existence of a grating at zero bias can be ex-

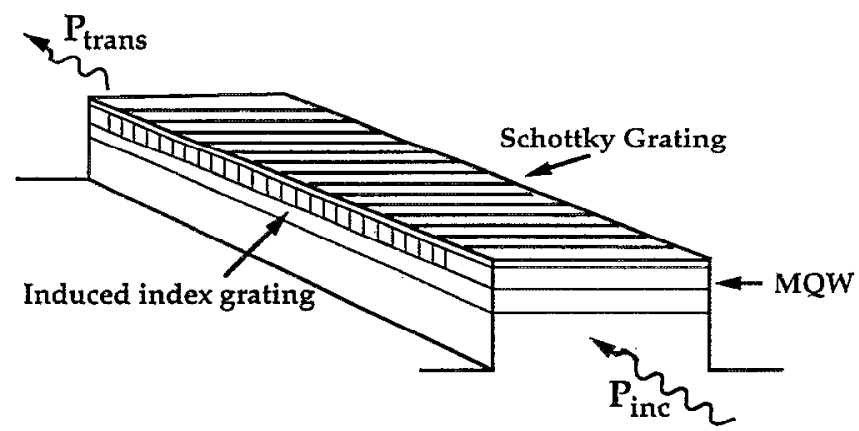

(a)



(b)

FIG. 2. (a) Waveguide shown schematically. (b) SEM photograph of waveguide and grating, airbridged to contact pad, before subsequent oxide deposition.

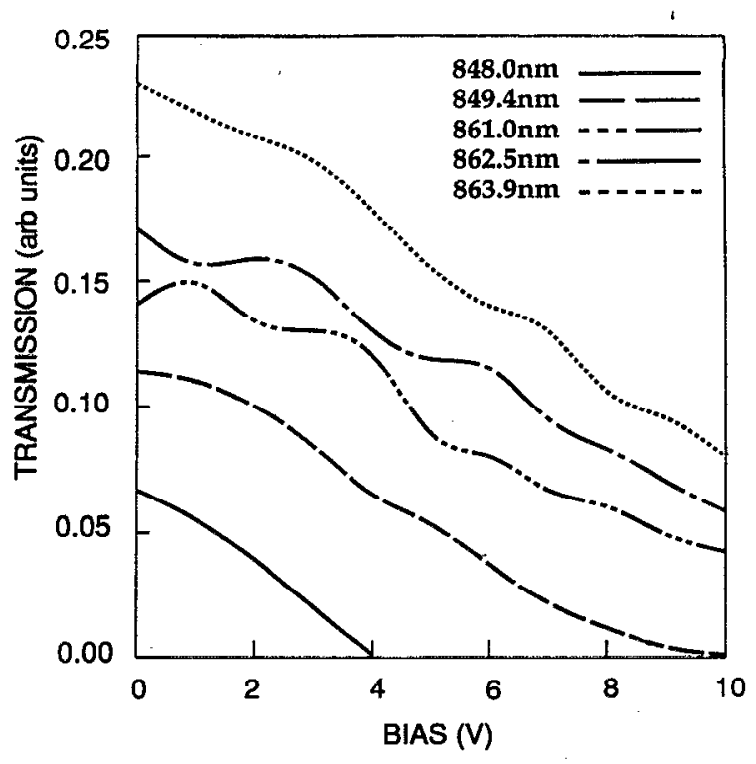

FIG. 3. Transmission of guided light showing electroabsorption at different wavelengths.

plained by a $0.9 \mathrm{eV}$ Schottky barrier height that can usually be formed on $\mathrm{Al}_{0.3} \mathrm{Ga}_{0.7} \mathrm{As}$. As is the case with III-V compound semiconductors, even a semiconductor-air interface has a potential barrier height roughly equal to the corresponding metal semiconductor contact barrier height, due to surface states on an unpassivated surface and the associated Fermi level pinning. This fact necessitated a PECVD $\mathrm{SiO}_{2}$ passivating layer to reduce the off-finger surface potential and preserve a zero-bias field-induced index grating. For a reverse bias of $-1 \mathrm{~V}$, the filter resonance shifts by $4 \AA$ to a longer wavelength. At $-2 \mathrm{~V}$, the peak shifts another $4 \AA$. At $-4 \mathrm{~V}$ applied bias, we gradually enter the realm of signifi-

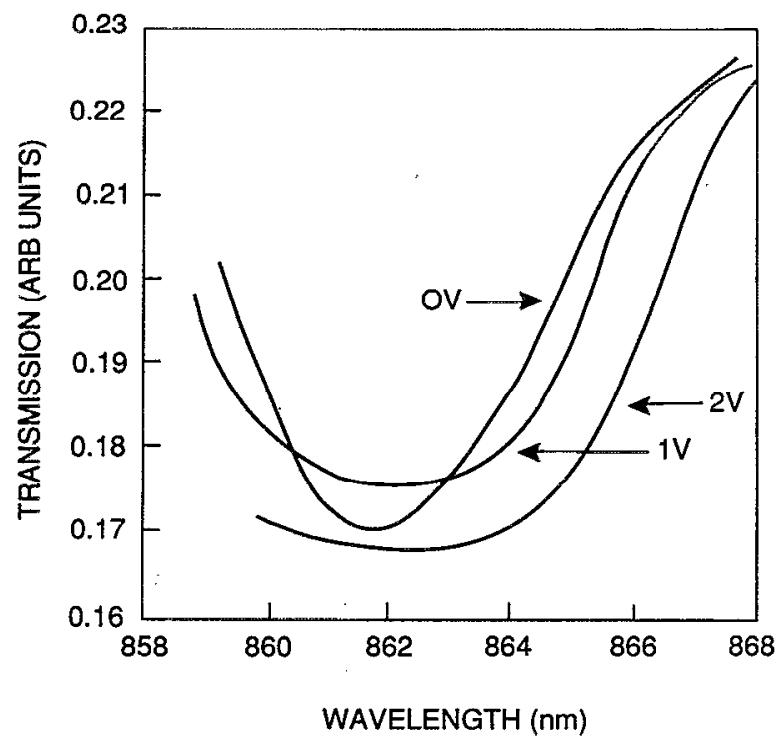

FIG. 4. Transmission spectra of filter for $0,-1,-2 \mathrm{~V}$ applied, showing DBF filtering resonances both broadening and shifting to longer wavelengths with increased bias. 
cant absorption, and filtering action is eclipsed by the larger effect of signal attenuation. At yet higher reverse biases, the guided wave becomes virtually extinct and the device resembles an ordinary $E-O$ waveguide modulator. In addition, due to fringing fields at increasing biases, the index grating gets increasingly unresolved. This results in resonant peak broadening for these higher biases. This effect too, is, readily apparent from the given transmission data.

The dip in the transmission intensity curve is not to be confused with a similar dip associated with the heavy-hole absorption peak. It is critically important to be able to separate the effects of electroabsorption and the filtering action in the presented data. The resonance due to absorption, while it has a similar field dependence, would occur at $845 \mathrm{~nm}$ (the location of the heavy-hole absorption peak). It is naturally desirable to operate well away from this wavelength. In the presented data, all three curves are shown to have a dip above $860 \mathrm{~nm}$. While one may look at the positive slope of these curves to the left of the resonance and skeptically attribute it to absorption, one cannot reconcile the negative slope to the left of the resonance unless filtering is the dominating mechanism.

In conclusion, we have developed a novel tunable filtering device with possible applications in the realm of optical communications and signal processing. By the application of $-2 \mathrm{~V}$ bias on this MQS-DBR waveguide, we were able to shift the Bragg resonance by $8 \AA$, thus demonstrating an integrable tunable filter. Through more exacting optimization, lithography calibration and longer written grating fields. devices with greater tunability, narrower band passing, and stronger filtering may be obtained.

The authors wish thank Dr. H.-C. Sun and Dr. Y.-C. Chen for the MBE growth of this device structure. This work is supported by the Air Force Office for Scientific Research under Grants AFOSR-91-0434 and F49620-92-J-0304-DEF.

${ }^{1} \mathrm{H}$. Haus, Waves and Fields in Optoelectronics (Prentice Hall, Englewood, NJ, 1984).

${ }^{2}$ M. Nakamura, K. Aiki, J. Umeda, and A. Yariv, Appl. Phys. Lett. 27 (1975).

${ }^{3}$ S. Noda, K. Kojima, K. Mitsunaga, K. Kyuma, K. Hamanaka, and T. Nakayama, IEEE J. Quantum Electron. QE-23 (1987).

${ }^{4}$ J. G. Mendoza-Alvarez, F. D. Nunes, and N. B. Patel, J. Appl. Phys. 51, 4365 (1980).

${ }^{5}$ Y. Kotaki and H. Ishikawa, IEE Proc. J. 138, 171 (1991).

${ }^{6}$ D. A. B. Miller, D. S. Chemla, T. C. Damen, A. C. Gossard, W. Wiegmann, T. H. Wood, and C. A. Burrus, Phys. Rev. Lett. 53, 2173 (1984).

${ }^{7}$ T. H. Wood, C. A. Burrus, D. A. B. Miller, D. S. Chemla, T. C. Damen, A. C. Gossard, and W. Wiegman, Appl. Phys. Lett. 44, 16 (1984).

${ }^{8}$ S. Goswami, S.-C. Hong, D. Biswas, P. K. Bhattacharya, J. Singh, and W.-Q. Li, IEEE J. Quantum Electron. 27, 760 (1991).

${ }^{9}$ J. S. Weincr, D. A. B. Miller, and D. Chemla, Appl. Phys. Lett. 50, 842 (1987).

${ }^{10}$ D. A. B. Miller, J. S. Weiner, and D. S. Chemla, IEEE J. Quantum Electron. QE-22, 1816 (1986) 